\title{
How obedience of marriage rules may counteract genetic drift
}

\author{
Michael Krawczak • Robert H. Barnes
}

Received: 16 November 2009 / Accepted: 13 January 2010/Published online: 21 February 2010

(C) Springer-Verlag 2010

\begin{abstract}
Marriage rules are a common component of many human societies. Since these rules, translated into mating patterns, would imply inbreeding, the question arises as to their long-term population genetic effects. We show by simulation that continuous unilateral or bilateral cross-cousin mating, reflecting the most common form of prescribed marriage, increases homozygosity but at the same time slows down considerably the loss of gene diversity due to genetic drift. For X-chromosomal genes, this effect is more pronounced if marriage, translated into mating, is matrilateral rather than patrilateral. Although the maintenance of gene diversity, in principle, could have conferred a selective advantage to the initiation of marriage rules, the mechanisms driving such a move are difficult to perceive. We therefore conclude that the possible preservation of gene diversity through marriage rule-induced inbreeding is a by-product, not the source, of a cultural invention that instead rested on foresight and strategic thinking.
\end{abstract}

Keywords Marriage rule · Population genetics · Inbreeding • Genetic drift · Social evolution · Gene diversity

M. Krawczak $(\bowtie)$

Institut für Medizinische Informatik und Statistik,

Christian-Albrechts-Universität zu Kiel,

Brunswiker Straße 10,

24105 Kiel, Germany

e-mail: krawczak@medinfo.uni-kiel.de

R. H. Barnes

Institute of Social and Cultural Anthropology,

University of Oxford,

51/53 Banbury Road,

Oxford OX2 6PE, UK

\section{Introduction}

In a number of indigenous human societies, including those of South India and Sri Lanka, Indonesia, Burma, Eastern Siberia, Australia, lowland South-America and possibly Northern Canada, marriage has been, and still is, governed by certain positive rules ${ }^{1}$ that actively prescribe marriage into specific categories of relatives (Dumont 1957; LéviStrauss 1969; Leach 1951; Needham 1958, 1962; Trautmann 2009). Although the respective categories are socially rather than biologically (i.e., genealogically) defined, which means that several types of genealogical relatives will fall into the relevant social category and will thus be marriageable, certain genealogical relatives can still be said to be typical of the category in question (Dumont 1968; Needham 1962). It is therefore common practice among social anthropologists to speak of the rules in this way, leading to the use of expressions such as "cross-cousin marriage," "mother's brother's daughter marriage," and so on.

Marriage rules clearly are not the same thing as mating rules. However, the assumption that marriage generally superimposes mating (i.e., reproductive) restrictions, at least upon the female member(s), and that males are also rarely free to reproduce much outside their marriage(s), would imply that marriage and mating may be correlated in societies that closely conform to positive marriage rules. This raises the question as to the possible population genetic consequences of marriage rules because virtually all of the rules in question would promote consanguineous mating, i.e., inbreeding.

Inbreeding is known to increase homozygosity in populations owing to the consequent increase in identical-

\footnotetext{
${ }^{1}$ Positive marriage rules are the opposite of negative marriage rules, which only specify relatives who may not be married.
} 
by-descent probability (Bittles 2005). Therefore, the incidence of recessive diseases can be expected to be positively correlated with the level of inbreeding induced by a particular marriage rule, translated into mating pattern. Finite population size also increases homozygosity through genetic drift, even if mating is at random, and this relationship has occasionally been referred to as the "parallelism between inbreeding and genetic drift" (Hamilton 2009). However, while inbreeding does not affect genetic diversity per se (even the most extreme form of inbreeding, namely selfing, leaves allele frequencies unchanged in an infinitely large population), genetic drift inevitably leads to the preponderance (and ultimate fixation, if mutation is lacking) of one allele per variant site. What is less clear, in our view, is how inbreeding and genetic drift would interact in small populations practicing marriage rules, translated into mating patterns. We therefore set out to study, by simulation, the temporal development of homozygosity and genetic diversity in finite populations of various sizes, each time assuming continued practice of consanguineous mating according to one of the three most commonly discussed types of marriage rule (Dumont 1968; Needham 1962).

The marriage rules considered here are commonly described as

1. "matrilateral cross-cousin marriage," in which a man marries his mother's brother's daughter, while a woman marries her father's sister's son (Fig. 1a), and

2. "patrilateral cross-cousin marriage," in which a man marries his father's sister's daughter, while a woman marries her mother's brother's son (Fig. 1b).

In addition to these "asymmetric" marriage rules, some human societies practice a symmetric marriage rule called

3. "bilateral cross-cousin marriage," in which a man marries a women who is simultaneously his mother's brother's daughter and his father's sister's daughter, while a woman marriages a man who is both her father's sister's son and her mother's brother's son (Fig. 1c). Bilateral crosscousin marriage entails sister exchange or, from the woman's point of view, brother exchange.

Both, contingent demographic and contingent social factors militate against it being possible, for example, for every male in a society being able to marry his genealogical mother's brother's daughter. The latter would be a limiting case as opposed to the actual situation prevailing in even very small communities. The assumption, however, that there were $100 \%$ conformity to a marriage rule interpreted as a mating rule led us to surprising results which we think are worth contemplating. Furthermore, the actual genetic consequences of a given marriage rule can be assumed to lie somewhere in between the limiting case described above and the pursuit of random mating, scenarios that we have

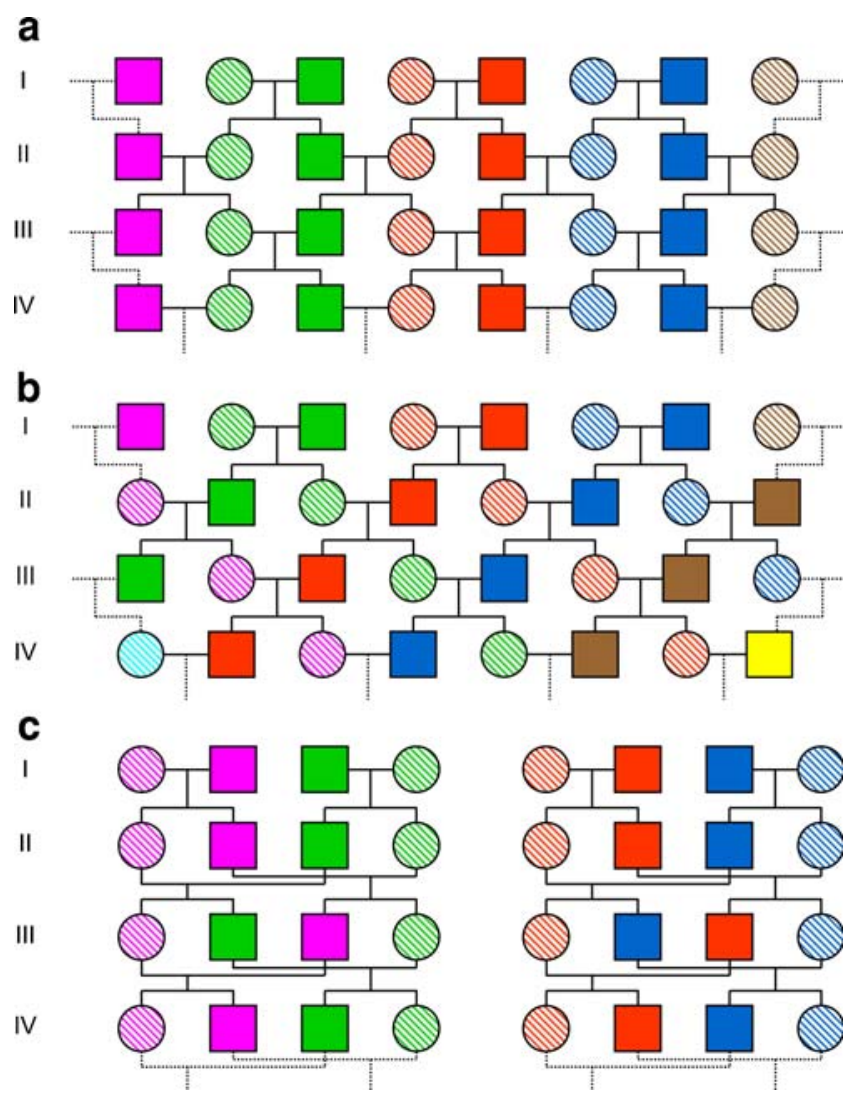

Fig. 1 Graphical representation on an idealized practice of different types of marriage, involving cross-cousin marriage. Circles indicate females; squares indicate males; different coloring is used to identify prevalent matrilines (hatched symbols) and patrilines (solid symbols)

considered in our simulations as reflecting the opposite ends of one and the same scale.

\section{Methods}

The possible population genetic effects of the two asymmetric types of marriage rule, i.e., matrilateral and patrilateral cross-cousin marriage, were assessed by simulation. To this end, we made the simplifying assumption that in a population of constant size $\mathrm{N}$, all mating produced exactly one male and one female offspring. Starting from a founder population of unrelated, randomly mating individuals (i.e., $\mathrm{N} / 2$ females and N/2 male) and their offspring, we assumed that all subsequent matings were either between a man and his father's sister's daughter (patrilateral) or his mother's brother's daughter (matrilateral), respectively. For comparison, we also simulated matings between randomly formed pairs of males and females (deliberately avoiding incestuous brother-sister matings). We did not simulate matings according to the symmetric rule of bilateral cross-cousin marriage. This is because strict obedience of this rule would create N/4 demes, each of size four, that would very rapidly 
drift towards fixation of one of the initially present alleles (see below).

We considered a single gene for which all alleles initially present in the population were different. The population genetic effects of different marriage rules, translated into mating patterns, were then measured by the "gene diversity half life" $t_{1 / 2}(h)$, i.e., the number of generations until the "gene diversity," defined as the expected heterozygosity under Hardy-Weinberg conditions,

$h=1-\sum_{i} f_{i}^{2}$

has fallen from $100 \%$ (its starting value; see above) to $50 \%$. Here, $f_{i}$ denotes the respective population frequency of the ith allele. The degree of inbreeding at $t_{1 / 2}(h)$ was assessed by recording the actually observed proportion of heterozygotes in the simulated population (or "observed heterozygosity," for short) at that time point.

Simulations were carried out 1,000 times, each time adopting a different population size ( $N=10$ to 150 , in steps of ten). We also distinguished between autosomal and $\mathrm{X}$ chromosomal genes since the laterality of cousin matings would only matter genetically for sex-linked genes. We did not consider Y-chromosomal genes, however, because the allelic spectrum of such genes would remain unchanged under the idealized conditions assumed here (namely one male offspring per male population member per generation).

\section{Results}

For both, autosomal and X-chromosomal genes, our simulations revealed that the gene diversity half life is much longer in populations pursuing (highly idealized) cross-cousin mating than in randomly mating populations (Fig. 2). In a population of 100 individuals (50 females, 50 males), for example, the median half life of autosomal gene diversity was 250 generations assuming random mating, compared to 644 generations for cross-cousin matings (Fig. 2a). If the population size equalled 150, the corresponding figures were 378 and 1,283, respectively. The difference was even more pronounced for X-chromosomal genes (Fig. 2b). For a population of size $N=100$, the gene diversity half life under matrilateral cross-cousin mating was approximately six times that under random mating $(1,616$ generations vs 278 generations), and still four times higher than with patrilateral cross-cousin mating (416 generations). If the population size increased to $N=150$, the corresponding figures were 426 (random), 715 (patrilateral), and 3,440 generations (matrilateral), respectively.

Not surprisingly, the lower rate of gene diversity decline in populations pursuing cross-cousin mating was accompanied by a higher level of inbreeding (formally defined here as the amount of heterozygote deficit relative to HardyWeinberg expectation). For $N=100$, the mean observed heterozygosity at $t_{1 / 2}(h)$, i.e., the time when the expected heterozygosity under Hardy-Weinberg conditions reached 0.5 , was $16 \%$ for autosomal genes, decreasing to $13 \%$ with $N=150$ (Fig. 3a). In the population practicing random mating, the mean observed heterozygosity at $t_{1 / 2}(h)$ equalled approximately $50 \%$, as expected, independent of the population size (Fig. 3a). For X-chromosomal genes, the level of inbreeding was notably higher with matrilateral than with patrilateral cross-cousin mating (Fig. 3b). If $N=$ 100 , the observed heterozygosity at $t_{1 / 2}(h)$ was $32 \%$ for patrilateral and $7 \%$ for matrilateral cross-cousin mating, decreasing to $26 \%$ and $5 \%$, respectively, for $N=150$.

As pointed out above, no simulations were performed assuming bilateral cross-cousin mating. Under the highly idealized assumptions made here, each deme would very rapidly drift towards fixation. In fact, Wright (1921) has shown that the expected proportion $p(t)$ of heterozygotes at time $\mathrm{t}$ fulfills the recursive relationship

$p(t)=p(t-1)-\frac{p(t-4)}{16}$

where $p(1)=p(2)=p(3)=1$ and $p(4)=7 / 8$ in our case. This series continues as 13/16 $(t=5), 24 / 32 \quad(t=6)$, and 44/64 $(t=7)$. It first falls below $1 / 2$ and $1 / 4$ at $t=11$ and $t=20$, respectively. At $t=57, p(t)=0.0096$ as the first value smaller than 0.01 which means that the probability of still observing at least one heterozygote in the deme is smaller than $1 \%$ after 57 or more generations of bilateral crosscousin mating. Further, since all alleles in the starting generation were assumed to be different in our simulations, each deme would become fixed for a different allele. Therefore, the gene diversity in the population as a whole eventually becomes

$h_{\text {fixation }}=1-\sum_{i} f_{i}^{2}=1-\frac{N}{4} \cdot\left(\frac{4}{N}\right)^{2}=\frac{N-4}{N}$

which exceeds 0.5 for $N>8$.

For the sake of simplicity, we have also chosen not to consider mutation or selection in our simulations, both of which are known to influence the genetic structure of populations in various ways. While recurrent mutation and balancing selection offset genetic drift, directional selection accelerates the process of fixation of advantageous alleles. However, since inbreeding speeds up the removal of deleterious mutations from a given population even further (Gillespie 1998), we may surmise that the only way in which the gene diversity-preserving effect of marriage ruleinduced inbreeding would be impeded by directional selection is in terms of a more rapid loss of rare recessive mutations. 
Fig. 2 Marriage rule-induced relationship between population size $N$ and gene diversity half life $t_{1 / 2}(h)$ for autosomal (a) and $\mathrm{X}$-chromosomal (b) genes. Displayed are the median and percentiles of $t_{1 / 2}(h)$ as obtained from 1,000 simulations per combination of marriage rule and population size. Note that, for better resolution, the vertical axes in the two component figures have been chosen to scale differently. a Black line: cross-cousin mating, red lines: random mating, and gray area and faint lines: upper and lower fifth percentile of $t_{1 / 2}(h)$. b Black line: matrilateral cross-cousin mating, blue lines: patrilateral cross-cousin mating, red lines: random mating, and gray area and faint lines: upper and lower fifth percentile of $t_{1 / 2}(h)$
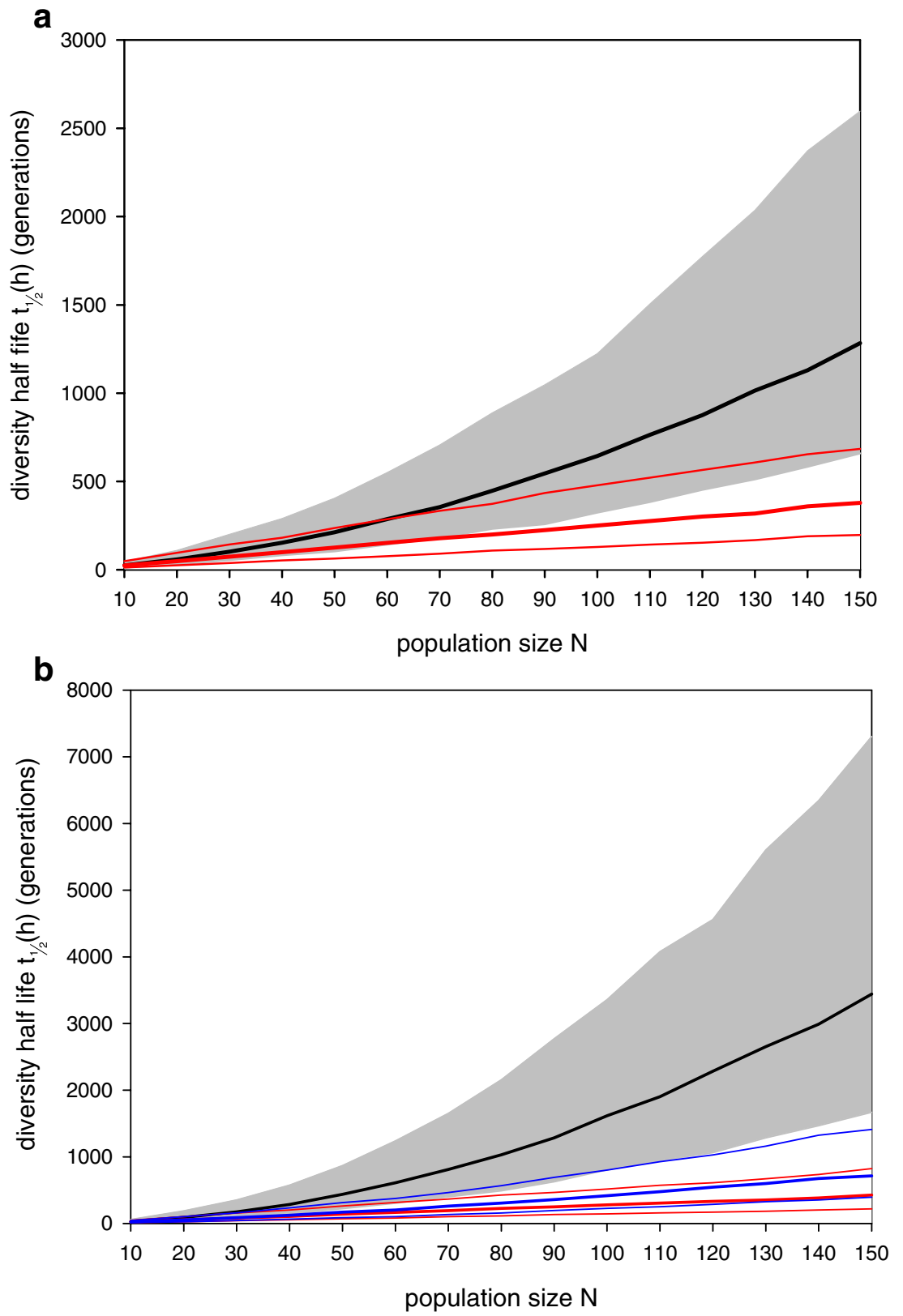

\section{Discussion}

Our simulation study has revealed that continued consanguineous mating according to particular marriage rules is capable of counteracting genetic drift in small populations. This actuality became most obvious for bilateral crosscousin mating, which essentially structures a population into small demes. Within each deme, genetic drift very rapidly leads to fixation, and we observed that, after less than 60 generations of strict obedience of the rule, more than $99 \%$ of all demes will have lost their internal diversity at a given locus. At the same time, the overall gene diversity in the population as a whole will equal $(N-4) / N$, a ratio that is close to unity even for modestly large values of
N. Strict bilateral cross-cousin mating does of course represent an extreme case of inbreeding in that it turns the population undergoing it into a conglomerate of tiny isolates. However, a similar gene diversity-preserving effect was observed for unilateral cross-cousin mating, which does not structure a population at all. After a sufficiently large number of generations of idealized practice of this mating pattern, the set of genealogical ancestors of every extant population member will comprise the whole starting population. This notwithstanding gene diversity declines much less rapidly in populations practicing unilateral crosscousin mating than in random mating populations. For Xchromosomal genes, the delay in the loss of the gene diversity was even more pronounced for matrilateral than 

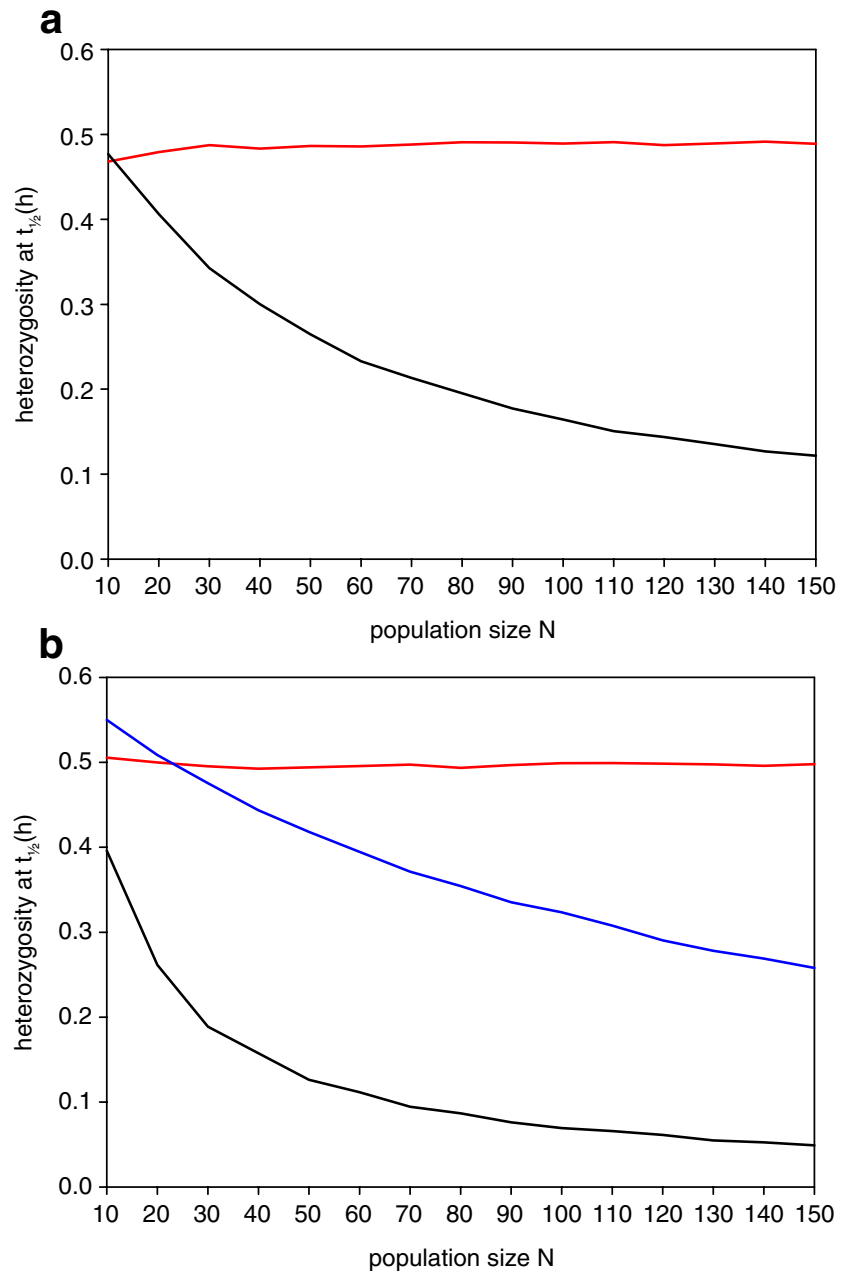

Fig. 3 Marriage rule-induced relationship between population size $N$ and the observed heterozygosity at gene diversity half life $t_{1 / 2}(h)$ for autosomal (a) and X-chromosomal (b) genes. Displayed is the mean observed heterozygosity as obtained from 1,000 simulations per combination of marriage rule and population size. a Black line: cross-cousin mating, red line: random mating. b Black line: matrilateral cross-cousin mating; blue line: patrilateral cross-cousin mating, red line: random mating

for patrilateral mating. Interestingly, matrilateral cross-cousin marriage is the much more prevalent type of unilateral crosscousin marriage, and both Lévi-Strauss (1969) and Needham (1958) have argued that patrilateral cross-cousin marriage empirically occurs nowhere as a total system.

As was to be expected, inbreeding in the form of crosscousin mating increases the level of homozygosity which means that a greater reduction in fitness compared to random mating can be expected to arise, owing to the presence of recessive disease genes (Bittles 2005). However, if marriage indeed correlates sufficiently well with mating in populations practicing marriage rules, the benefits arising from these rules must have outweighed their detrimental consequences for the rules to have become evolutionarily stable (a paradigm enshrined in "Hamilton's Rule"; Hamilton
1970). Such balancing would appear possible, for example, if marriage alliances and the practicing of marriage rules served to increase the efficiency with which the available resources were used by the respective societies to such an extent that coping with cases of recessive diseases no longer impeded people's inclusive reproductive fitness too much. What remains unclear, however, is whether the prolonged maintenance of overall gene diversity due to marriage ruleinduced inbreeding was a mere side effect of an otherwise selected practice or whether it may have represented an independent target of evolutionary pressure towards the initiation of prescribed consanguineous mating.

Maintenance of gene diversity is particularly advantageous in rapidly changing environments. Although we have assumed in our simplified simulations that all individuals reproduced equally well, at least some selection will have occurred in any real populations of interest in the past. Under such circumstances, the presence of greater gene diversity provides greater flexibility and more degrees of freedom for the population to move on an existing adaptive landscape or to approach new adaptive peaks on a changing landscape (Wright 1988). Although evolution may thus have been capable to "see" the overall gene diversity of a population, however, we still find it difficult to perceive mechanisms by which this evolutionary pressure may have led to the invention and implementation of marriage rules. Accepting that ancient societies had no understanding of genetics, the only way to link controlled mating to higher fitness would have been by trial-and-error or empirical observation. Both of these scenarios appear unlikely, however, because marriage rules are a very complex cultural entity that is neither easy to infer from animal breeding experience nor likely to arise from social experimentation. Furthermore, although consanguineous mating may have been frequent in prehistoric populations of Homo sapiens, this is most likely to have occurred as an enforced response to the small size and wide geographic dispersal of early hunter-gatherer communities (Bittles and Black 2010). We therefore conclude that the gene diversity-preserving effect of marriage rule-induced inbreeding was a by-product, not the major stimulus, of a cultural invention that instead rested on foresight and strategic thinking. Our findings nevertheless lend additional support to Vogel and Motulsky (1997) who rejected public health measures against consanguineous mating on the ground that these rules are important components of social structures. They concluded that "attempts to break up marriage customs with long traditions [...] should not be initiated unless all possible social consequences have been carefully considered." From a genetic point of view, our results have shown that there is more to these customs: In small populations in particular, the obedience of marriage rules translated into mating patterns (potentially) counteracts the homogenizing effects of genetic drift. 
Acknowledgments The authors are most grateful to Jörg Schmidtke, Hannover, for the helpful discussion.

\section{References}

Bittles AH (2005) Endogamy, consanguinity and community disease profiles. Community Genet 8:17-20

Bittles AH, Black ML (2010) Evolution in Health and Medicine Sackler Colloquium: Consanguinity, human evolution, and complex diseases. Proc Natl Acad Sci USA 17:1779-1786

Dumont L (1957) Hierarchy and marriage alliance in South Indian kinship (occasional papers of the Royal Anthropological Institute of Great Britain and Ireland No. 12). Royal Anthropological Institute, London

Dumont L (1968) Marriage alliance. In: Sills D (ed) International encyclopedia of the social sciences, vol. 10. Macmillan \& Free Press, New York, pp 19-23

Gillespie JH (1998) Population genetics - a concise guide. The Johns Hopkins University Press, Baltimore, pp 90-96
Hamilton WD (1970) Selfish and spiteful behaviour in an evolutionary model. Nature 228:1218-1220

Hamilton MB (2009) Population genetics. Wiley-Blackwell, Chichester, pp 78-80

Leach ER (1951) The structural implications of matrilateral cross-cousin marriage. J R Anthropol Inst 81:23-55

Lévi-Strauss C (1969) The elementary structures of kinship. Bell JH, von Sturmer JR, Needham R (trans) Eyre \& Spottiswoode. London; Beacon Press, Boston

Needham R (1958) The formal analysis of prescriptive patrilateral cross-cousin marriage. Southwest J Anthropol 14:199-219

Needham R (1962) Structure and sentiment. University of Chicago Press, Chicago

Trautmann TR (2009) The clash of chronologies: ancient India in the modern world. Yoda Press, New Delhi

Vogel F, Motulsky A (1997) Human genetics: problems and approaches, 3rd edn. Springer, Berlin, p 559

Wright S (1921) Systems of mating II. The effects of inbreeding on the genetic composition of populations. Genetics 6:124-143

Wright S (1988) Surfaces of selective value revisited. Am Nat $131: 115-123$ 